\title{
DOUBLE VOGAN DIAGRAMS AND IRREDUCIBLE PSEUDO-HERMITIAN SYMMETRIC SPACES
}

\author{
Meng-Kiat Chuah
}

\begin{abstract}
Let $G$ be a real simple Lie group with Lie algebra $\mathfrak{g}$. We consider additional structures on the Dynkin diagram of the complexification of $\mathfrak{g}$, known as the double Vogan diagrams. They lead to a combinatorial classification of the pseudo-Hermitian symmetric spaces of $G$.
\end{abstract}

\section{Introduction}

Let $G$ be a real simple Lie group. Let $\sigma$ be an involution on $G$, and $G^{\sigma}$ be its invariant subgroup. We say that $G / G^{\sigma}$ is a symmetric space. If in addition it has a $G$-invariant pseudo-Hermitian structure, we say that it is pseudo-Hermitian. The symmetric spaces are classified by Berger [1], and the pseudo-Hermitian symmetric spaces are classified by Shapiro [7]. In [3], we introduce the double Vogan diagrams, which leads to another classification of the symmetric spaces. This paper provides the combinatorial conditions on these diagrams to obtain an easy classification of the irreducible pseudo-Hermitian symmetric spaces.

To avoid discussions on the topology of the symmetric spaces such as connectedness and coverings, we work with Lie algebras. So $\mathfrak{g}$ is a simple Lie algebra, $\sigma$ is a $\mathfrak{g}$ involution with invariant subalgebra $\mathfrak{g}^{\sigma}$, and $\left(\mathfrak{g}, \mathfrak{g}^{\sigma}\right)$ is called a symmetric pair. We consider only irreducible symmetric pairs, namely $\mathfrak{g}$ is a real form of a complex simple Lie algebra.

Let $L$ be a complex simple Lie algebra, and let $\mathrm{D}$ be its Dynkin diagram. A diagram symmetry on $\mathrm{D}$ is an automorphism $f: \mathrm{D} \longrightarrow \mathrm{D}$ preserving edge relations. Let $r=\operatorname{ord}(f)$ be its order. It leads to an affine diagram $\mathrm{D}^{r}$. We ignore $r=3$ for our purpose, so $\mathrm{D}^{r}$ is $\mathrm{D}^{1}$ for any $\mathrm{D}$, or $A_{n}^{2}, D_{n}^{2}, E_{6}^{2}$. The vertices $\alpha \in \mathrm{D}^{r}$ represent roots of $L$, and there are canonical positive integers $m_{\alpha}$ without non-trivial common factor such that $\sum_{\mathrm{D}^{r}} m_{\alpha} \alpha=0[4, \mathrm{Ch} . \mathrm{X}-5][5$, Ch. 4$]$.

Received by the editors January 27, 2012.

2010 Mathematics Subject Classification. 17B20, 53C35.

Key words and phrases. pseudo-Hermitian symmetric space, real simple Lie algebra, Dynkin diagram. 
A painting on $\mathrm{D}^{r}$ is a choice of white or black color on each vertex. A circling on $\mathrm{D}^{r}$ is a choice of circling or not circling on each vertex. A double Vogan diagram is a pair $(p, c)$ on $\mathrm{D}^{r}$, where $p$ is a painting and $c$ is a circling such that

$$
r \sum_{\text {black }} m_{\alpha}=2 \text { and } r \sum_{\text {circled }} m_{\alpha} \text { is even. }
$$

Then $p$ represents a real form $\mathfrak{g}$ of $L$, and $c$ represents a $\mathfrak{g}$-involution $\sigma$, so that $(p, c)$ represents a symmetric pair $\left(\mathfrak{g}, \mathfrak{g}^{\sigma}\right)$ in the sense of [3, Def. 1.2, Thm. 1.3] (see also the explanation after (2.1)).

By $p$, the subdiagram of white vertices is the Dynkin diagram of a maximally compact subalgebra $\mathfrak{k}$ of $\mathfrak{g}$, so we denote it by $\mathrm{D}_{\mathfrak{k}} \subset \mathrm{D}^{r}$. If $C$ is a connected component of $\mathrm{D}_{\mathfrak{k}}$, we define positive integers $n_{\alpha}$ for all $\alpha \in C$ so that $\sum_{C} n_{\alpha} \alpha$ is the highest root of $C$. In Definition 2.6, we define an equivalence relation on the set of all circlings on $\mathrm{D}^{r}$. The following is the main theorem of this article.

Theorem 1.1. Every irreducible pseudo-Hermitian symmetric pair can be represented by a double Vogan diagram $(p, c)$ on $\mathrm{D}^{r}$ such that:

(a) For $r=1, c$ is equivalent to a circling of two vertices $\{\alpha, \beta\}$ where $m_{\alpha}=$ $m_{\beta}=1$.

(b) For $r=2$, c circles two vertices $\{\alpha, \beta\}$ where $\alpha$ is white, $m_{\alpha}=n_{\alpha}=1$ and $\beta$ is black.

Conversely, a double Vogan diagram that satisfies (a) or (b) represents an irreducible pseudo-Hermitian symmetric pair.

In this theorem, $\mathfrak{g}^{\sigma}$ is unique up to its conjugation class by aut $(\mathfrak{g})$. We prove Theorem 1.1 in Section 2. It provides a combinatorial method to classify the pseudoHermitian symmetric pairs. In Section 3, we give some examples to demonstrate this method. In Section 4, we give a complete list of pseudo-Hermitian symmetric pairs along with their double Vogan diagrams.

\section{Pseudo-Hermitian symmetric pairs}

In this section, we prove Theorem 1.1. Recall that $L$ is a complex simple Lie algebra, $\mathrm{D}$ is its Dynkin diagram, $\mathrm{D}^{r}$ is the affine diagram obtained from a diagram symmetry on $\mathrm{D}$ of order $r$, and the canonical integers $m_{\alpha}$ satisfy $\sum_{\mathrm{D}^{r}} m_{\alpha} \alpha=0$.

A Kac diagram is a painting on $\mathrm{D}^{r}$ such that $r \sum_{\text {black }} m_{\alpha}=2$. A Vogan diagram is a pair $(c, d)$ on $\mathrm{D}$, where $d$ is a diagram involution on $\mathrm{D}$, and $c$ is a circling on the vertices fixed by $d$. In $[6$, Ch.VI-8], the Vogan diagrams are shown to represent $L$-involutions. 
Theorem 2.1. A Kac diagram or Vogan diagram represents an L-involution $\sigma$, and every L-involution is represented by a Kac diagram and a Vogan diagram.

(a) For the Kac diagram, we have $L^{\sigma}=L_{\mathrm{ss}}^{\sigma}+Z$, where $L_{\mathrm{ss}}^{\sigma}=\left[L^{\sigma}, L^{\sigma}\right]$ has Dynkin diagram $\{$ white vertices $\}$ and the center $Z$ of $L^{\sigma}$ has dimension $\sharp\{$ black vertices $\}-1$.

(b) For the Vogan diagram $(c, d)$, if $d \neq 1$, then $L^{\sigma}$ is semisimple.

(c) The above assertions about Vogan diagrams remain valid if $L=L^{\prime} \oplus L^{\prime}$ where $L^{\prime}$ is complex simple, and d permutes the two connected components of $\mathrm{D}$.

Proof. The assertions about Kac diagrams are proved in [5, Ch.8]. For part (b), by [6, Thm.6.88], every Vogan diagram represents an $L$-involution $\sigma$. The $L$-involution $\sigma$ is represented by a Vogan diagram with $d \neq 1$ if and only if it is represented by a Kac diagram with $r=2$. For $r=2$, there is only one black vertex on the Kac diagram, so $Z=0$, namely $L^{\sigma}$ is semisimple.

For part (c), $L^{\prime}$ (regarded as real) is a real form of $L$ with Vogan diagram $(1, d)$. Also, $L^{\prime}$ (regarded as complex) has a compact real form $\mathfrak{k}$ such that $L^{\prime}=\mathfrak{k}+i \mathfrak{k}$, and $\sigma$ restricts to a Cartan involution on $L^{\prime}$ such that $\left(L^{\prime}\right)^{\sigma}=\mathfrak{k}$. Then $L^{\sigma}=\mathfrak{k} \oplus \mathfrak{k}$ is semisimple. An example is $L^{\prime}=\mathfrak{s l}(n, \mathbb{C}), L=\mathfrak{s l}(n, \mathbb{C}) \oplus \mathfrak{s l}(n, \mathbb{C})$ and $L^{\sigma}=\mathfrak{s u}(n) \oplus$ $\mathfrak{s u}(n)$.

We combine the Kac and Vogan diagrams to write $(p, c, d)$ on $\mathrm{D}^{r}$, where $p$ is a Kac diagram and $(c, d)$ is a Vogan diagram. Namely $p$ is a painting on $\mathrm{D}^{r}, d$ is a $p$-stable diagram involution on $\mathrm{D}^{r}$, and $c$ is a circling on the vertices fixed by $d$. Define

$\mathcal{O}=\{$ circled vertices $\} \cup\{d$-orbits consisting of two adjacent vertices $\}$.

If $x=\{\alpha, \beta\} \in \mathcal{O}$ is a $d$-orbit, then $m_{\alpha}=m_{\beta}$, so we can define $m_{x}$ to be this integer. We say that $(p, c, d)$ is a double Vogan diagram if

$$
r \sum_{\text {black }} m_{\alpha}=2 \text { and } r \sum_{\mathcal{O}} m_{x} \text { is even. }
$$

It is said to represent a symmetric pair $\left(\mathfrak{g}, \mathfrak{g}^{\sigma}\right)$ if it satisfies [3, Def.1.2]. Namely $p$ represents an $L$-involution $\theta$, or equivalently a real form $\mathfrak{g}$ of $L$ where $\theta$ restricts to a Cartan involution on $\mathfrak{g}$. Then $(c, d)$ represents an $L$-involution $\sigma$ which commutes with $\theta$, or equivalently a $\mathfrak{g}$-involution $\sigma$. We identify the symmetric pair $\left(\mathfrak{g}, \mathfrak{g}^{\sigma}\right)$ with the commuting $L$-involutions $(\theta, \sigma)$.

Theorem 2.2. [3, Thm.1.3] Every double Vogan diagram uniquely represents a symmetric pair up to equivalence. Conversely, every symmetric pair is represented by a double Vogan diagram. 
Recall the following definition of pseudo-Hermitian symmetric pairs. We intend to find out the double Vogan diagrams which represent them.

Definition 2.3. $\quad$ [7, p.532] A symmetric pair $\left(\mathfrak{g}, \mathfrak{g}^{\sigma}\right)$ is called pseudo-Hermitian if $\mathfrak{g}^{\sigma}$ has a compact one-dimensional center.

We shall see in Corollary 2.5 that the double Vogan diagrams $(p, c, d)$ which represent pseudo-Hermitian symmetric pairs necessarily satisfy $d=1$, so that (2.1) simplifies to (1.1).

The next proposition and corollary provide some necessary conditions for pseudoHermitian symmetric pairs. In Proposition 2.4(b,c), we say that $d$ is non-trivial on each connected component of $\mathrm{D}_{\mathfrak{k}}$ if each connected component contains a vertex $\alpha$ such that $\alpha \neq d \alpha$. Thus either $d$ acts on each connected component non-trivially or permutes the connected components.

Proposition 2.4. Let $(p, c, d)$ be a double Vogan diagram on $\mathrm{D}^{r}$ which represents a symmetric pair. Each of the following conditions implies that the symmetric pair is not pseudo-Hermitian:

(a) $r=1, d$ is non-trivial and preserves $\mathrm{D} \subset \mathrm{D}^{1}$;

(b) $d$ is non-trivial on each connected component of $\mathrm{D}_{\mathfrak{k}}$, and there is only one black vertex;

(c) $d$ is non-trivial on each connected component of $\mathrm{D}_{\mathfrak{k}}$, and there are two black vertices switched by $d$.

Proof. Let $p$ represent a real form $\mathfrak{g}$ of $L$, or equivalently an $L$-involution $\theta$. Let $(c, d)$ represent a $\mathfrak{g}$-involution $\sigma$.

If $r=1, d$ is non-trivial and preserves $\mathrm{D} \subset \mathrm{D}^{1}$, then Theorem 2.1(b) says that $L^{\sigma}$ is semisimple, so $\mathfrak{g}^{\sigma}=\mathfrak{g} \cap L^{\sigma}$ is semisimple. By Definition 2.3, $\left(\mathfrak{g}, \mathfrak{g}^{\sigma}\right)$ is not pseudo-Hermitian. This proves part (a).

To prove parts (b) and (c), we now assume that $d$ is non-trivial on each connected component of $D_{\mathfrak{k}}$. Let $\mathfrak{g}=\mathfrak{k}+\mathfrak{p}$ be a Cartan decomposition, where $\theta= \pm 1$ on $\mathfrak{k}$ and $\mathfrak{p}$ respectively.

For part (b), since there is only one black vertex, $L^{\theta}$ is semisimple by Theorem 2.1(a). Write $L^{\theta}=\sum_{i} L_{i}^{\theta}$, where each $L_{i}^{\theta}$ is either simple or is of the form $L^{\prime} \oplus L^{\prime}$ such that $\sigma$ permutes the two copies of simple ideals $L^{\prime}$. By Theorem 2.1(b,c), each $\left(L_{i}^{\theta}\right)^{\sigma}$ is semisimple, so $\left(L^{\theta}\right)^{\sigma}=\sum_{i}\left(L_{i}^{\theta}\right)^{\sigma}$ is semisimple. Consequently, $\mathfrak{k}^{\sigma}=$ $\mathfrak{g} \cap\left(L^{\theta}\right)^{\sigma}$ is semisimple. By Definition 2.3, $\left(\mathfrak{g}, \mathfrak{g}^{\sigma}\right)$ is not pseudo-Hermitian. This proves part (b).

Finally consider part (c). Since there are two black vertices, by Theorem 2.1(a), we have $\mathfrak{k}=\mathfrak{k}_{\mathrm{ss}}+\mathfrak{z}$ where $\mathfrak{k}_{\mathrm{ss}}=[\mathfrak{k}, \mathfrak{k}]$ is semisimple and $\mathfrak{z}$ is a one-dimensional center of $\mathfrak{k}$. 
Similar to the argument for part (b), $\left(\mathfrak{k}_{\mathrm{ss}}\right)^{\sigma}$ is semisimple. Since $d$ switches the two black vertices, $\sigma=-1$ on $\mathfrak{z}\left[3\right.$, Prop.2.4(b)]. Hence $\mathfrak{k}^{\sigma}=\left(\mathfrak{k}_{\mathrm{ss}}\right)^{\sigma}$ is semisimple. Once again by Definition $2.3,\left(\mathfrak{g}, \mathfrak{g}^{\sigma}\right)$ is not pseudo-Hermitian. This proves part (c).

Corollary 2.5. Let $(p, c, d)$ be a double Vogan diagram which represents a symmetric pair. If $d \neq 1$, then the symmetric pair is not pseudo-Hermitian.

Proof. Let $(p, c, d)$ represent $\left(\mathfrak{g}, \mathfrak{g}^{\sigma}\right)$. We divide the arguments into two cases.

Case 1: $\mathfrak{g}=\mathfrak{s o}(p, q), p$ even, $q$ odd.

We treat this case separately because $B_{n}^{1}$ (where $n=\frac{1}{2}(p+q-1)$ ) has non-trivial diagram involution $d$ which does not satisfy any of the conditions in Proposition 2.4. Here $\mathfrak{g}^{\sigma}=\mathfrak{s o}(k, l)+\mathfrak{s o}(r, s)$, where $k+r=p$ and $l+s=q$. By Definition 2.3, $\mathfrak{s o}(p, q)$ has only two pseudo-Hermitian symmetric pairs, given by $\mathfrak{g}^{\sigma}=\mathfrak{s o}(p-2, q)+\mathfrak{s o}(2)$ and $\mathfrak{g}^{\sigma}=\mathfrak{s o}(p, q-2)+\mathfrak{s o}(2)$. They are given by Figure $4(6)$ of Section 4 (for $\mathfrak{s o}(p-2, q)+$ $\mathfrak{s o}(2)$, the $B$-end is closer to the circled black vertex than the circled white vertex; the opposite occurs for $\mathfrak{s o}(p, q-2)+\mathfrak{s o}(2))$. These diagrams satisfy definition (1.1) for double Vogan diagrams. Since they are the only two pseudo-Hermitian symmetric pairs, all other diagrams (in particular the ones with $d \neq 1$ ) do not represent pseudoHermitian symmetric pairs. This proves Case 1.

Case 2: All remaining $\mathfrak{g}$.

We check all the Kac diagrams [3, p.1740-1747]. For $\mathfrak{g}$ not in Case 1 , if $d \neq 1$, we see that $d$ satisfies one of the three situations (a), (b), (c) of Proposition 2.4. For example if $\mathfrak{g}=\mathfrak{e}_{6(-14)}$, then $d$ satisfies situation (c); and if $\mathfrak{g}=\mathfrak{e}_{6(2)}$, then $d$ satisfies situation (a) [3, p. 1743]. The corollary then follows from Proposition 2.4.

By the above corollary, we can assume $d=1$ on the double Vogan diagrams. Thus (2.1) simplifies to (1.1). Recall the following definition and theorem from [3].

Definition 2.6. Let $c$ be a circling on $\mathrm{D}^{r}$. If $\alpha$ is a circled vertex, define another circling $F_{\alpha} c$ by

$$
F_{\alpha}:\left\{\begin{array}{l}
\text { reverse circling conditions of all vertices } \beta \text { adjacent to } \alpha, \\
\text { unless } \beta \text { is a longer root joint to } \alpha \text { by a double edge. }
\end{array}\right.
$$

We say that two circlings $c, c^{\prime}$ are equivalent if and only if there is a sequence of circlings $c=c_{0} \rightarrow c_{1} \rightarrow \ldots \rightarrow c_{n}=c^{\prime}$ such that each $c_{i} \rightarrow c_{i+1}$ is given by some $F_{\alpha}$ of (2.2) or a diagram symmetry.

Theorem 2.7. [3, Thm.5.1] Let $c, c^{\prime}$ be circlings on $\mathrm{D}^{r}$ which represent L-involutions $\sigma, \sigma^{\prime}$ respectively. Then $c, c^{\prime}$ are equivalent if and only if $\sigma, \sigma^{\prime}$ are conjugate by an L-automorphism. 
Definition 2.6 and Theorem 2.7 provide a method to determine conjugate $L$ involutions. A systematic classification of equivalent circlings on $\mathrm{D}^{r}$ is given in [2].

\section{Proof of Theorem 1.1.}

The correspondence between double Vogan diagrams $(p, c, d)$ and symmetric pairs $\left(\mathfrak{g}, \mathfrak{g}^{\sigma}\right)$ is established in Theorem 2.2. Furthermore, Corollary 2.5 says that to consider pseudo-Hermitian symmetric pairs, we may assume that $d$ is trivial and work with $(p, c)$. We now show that the condition in Theorem 1.1(a,b) is necessary and sufficient for $\left(\mathfrak{g}, \mathfrak{g}^{\sigma}\right)$ to be pseudo-Hermitian.

We start with condition (a) of the theorem, namely $r=1$. By Theorems 2.1(a) and 2.7, $L^{\sigma}$ has a one-dimensional center $Z$ if and only if $c$ is equivalent to a circling of two vertices $\alpha, \beta$ such that $m_{\alpha}=m_{\beta}=1$. If this happens, $Z \cap \mathfrak{g}^{\sigma}$ is the center of $\mathfrak{g}^{\sigma}$. Since $r=1, \mathfrak{g}$ has a compact Cartan subalgebra, say $\mathfrak{t}$. Since $d=1, \sigma$ acts trivially on $\mathfrak{t}$, so $\mathfrak{t}$ is also a Cartan subalgebra of $\mathfrak{g}^{\sigma}$. A Cartan subalgebra automatically contains the center, so $\left(Z \cap \mathfrak{g}^{\sigma}\right) \subset \mathfrak{t}$, namely $Z \cap \mathfrak{g}^{\sigma}$ is compact. By Definition $2.3,\left(\mathfrak{g}, \mathfrak{g}^{\sigma}\right)$ is pseudo-Hermitian.

Next we consider condition (b) of the theorem, namely $r=2$. Here $p$ paints exactly one black vertex $\beta$, so $\mathrm{D}^{2}=\mathrm{D}_{\mathfrak{k}} \cup\{\beta\}$. Write $-\beta=\sum_{\mathrm{D}_{\mathfrak{k}}} n_{\alpha} \alpha$. Let $\mathfrak{g}=\mathfrak{k}+\mathfrak{p}$ be a $\sigma$-stable Cartan decomposition, and $\mathfrak{g}^{\sigma}=\mathfrak{k}^{\sigma}+\mathfrak{p}^{\sigma}$. By Theorem 2.1(a),

$$
\begin{aligned}
& \mathfrak{k}^{\sigma} \text { has a one-dimensional center } \mathfrak{z} \\
& \Longleftrightarrow \mathrm{D}_{\mathfrak{k}} \text { has a unique circled vertex } \alpha \text {, and } n_{\alpha}=1 .
\end{aligned}
$$

Suppose that the equivalent conditions in (2.3) hold. Let $\alpha$ be the unique circled vertex of $D_{\mathfrak{k}}$. We shall prove that

$$
\left[\mathfrak{p}^{\sigma}, \mathfrak{z}\right]=0 \Longleftrightarrow m_{\alpha}=1 \text { and } \beta \text { is circled, }
$$

so that Definition 2.3, (2.3) and (2.4) lead to Theorem 1.1(b).

Write $\mathrm{D}_{\mathfrak{k}} \backslash\{\alpha\}=\left\{\alpha_{i}\right\}$ and $m_{i}=m_{\alpha_{i}}$ for convenience. We claim that

$$
\beta+m_{\alpha} \alpha+\sum_{i} m_{i} \alpha_{i}=0, \quad \alpha_{i}(\mathfrak{z})=0, \quad \alpha(\mathfrak{z}) \neq 0 .
$$

The first condition of (2.5) follows from $m_{\beta}=1$. The second condition of (2.5) is due to the fact that $\alpha_{i}$ are the $\mathfrak{k}^{\sigma}$-roots (because $\left\{\alpha_{i}\right\}$ are not circled), and $\mathfrak{z}$ is the center of $\mathfrak{k}^{\sigma}$. The third condition of $(2.5)$ is due to the fact that $\mathfrak{k}$ is semisimple (because $r=2$ ), so some $\mathfrak{k}$-roots cannot annihilate $\mathfrak{z}$ and they have to include $\alpha$. This proves (2.5) as claimed.

We now prove (2.4). Suppose that $\left[\mathfrak{p}^{\sigma}, \mathfrak{z}\right]=0$. By $(2.5)$ we have $\beta(\mathfrak{z}) \neq 0$, so $\mathfrak{p}_{\beta} \not \subset \mathfrak{p}^{\sigma}$. Hence, vertex $\beta$ is circled. Since $\sigma=1$ on $\mathfrak{g}_{\alpha_{i}}$ and $\sigma=-1$ on $\mathfrak{g}_{\alpha_{i}}+\mathfrak{g}_{\beta}$, it follows from (2.5) that $m_{\alpha}$ is odd. Then the list of $\mathrm{D}^{2}$ shows that $m_{\alpha}=1,3$ [3, p. 1726]. Furthermore, $m_{\alpha}=3$ happens only on $E_{6}^{2}$, and in that case $n_{\alpha} \neq 1$, which contradicts

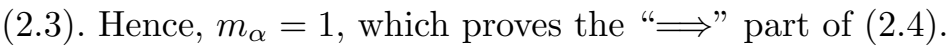


Conversely, suppose that $m_{\alpha}=1$ and $\beta$ is circled. Since $m_{\alpha}=1$, by $(2.5)$

$$
(\beta+\alpha)(\mathfrak{z})=0 .
$$

Since $\beta$ is the lowest root of $\mathfrak{p}$, and since $m_{\alpha}=1$, all $\mathfrak{p}$-roots are of the form

$$
\text { (a) } \beta+\sum_{i} c_{i} \alpha_{i}, \quad \text { (b) } \beta+\alpha+\sum_{i} c_{i} \alpha_{i} \text {. }
$$

Since $\sigma=-1$ on $\mathfrak{g}_{\beta}$ and $\sigma=1$ on $\mathfrak{g}_{\alpha_{i}}$, it follows that $\sigma=-1$ on the root spaces of $(2.7)(\mathrm{a})$. So the $\mathfrak{p}$ root spaces of $(2.7)(\mathrm{a})$ do not belong to $\mathfrak{p}^{\sigma}$. By the same reason, $\mathfrak{p}^{\sigma}$ consists of the $\mathfrak{p}$ root spaces of $(2.7)(\mathrm{b})$. By (2.5) and (2.6), we see that $(2.7)(\mathrm{b})$ annihilate $\mathfrak{z}$, so their $\mathfrak{p}$ root spaces commute with $\mathfrak{z}$. Hence $\left[\mathfrak{p}^{\sigma}, \mathfrak{z}\right]=0$. This proves the "£" part of (2.4). Part (b) of the theorem follows from Definition 2.3, (2.3) and (2.4). This completes the proof of Theorem 1.1.

\section{Examples}

In this section, we provide some examples. They illustrate the application of Theorem 1.1 to obtain irreducible pseudo-Hermitian symmetric pairs.

Example 3.1. Pseudo-Hermitian symmetric pair of $\mathfrak{s l}(2 n, \mathbb{R})$.

For $\mathfrak{g}=\mathfrak{s l}(2 n, \mathbb{R})$, the Kac diagram is given by Figure 1 (a) below. Recall that $\sum_{\mathrm{D}^{2}} m_{\alpha} \alpha=0$, and $\sum_{\mathrm{D}_{\mathfrak{k}}} n_{\alpha} \alpha$ is the highest root of $\mathrm{D}_{\mathfrak{k}}$. We indicate $\left(m_{\alpha}, n_{\alpha}\right)$ at the white vertices $\alpha \in \mathrm{D}_{\mathfrak{k}}$.

(a)

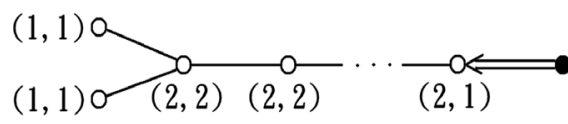

(b)

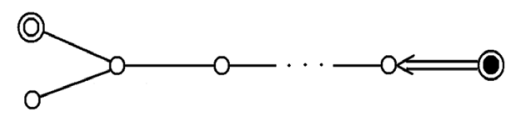

FiguRE 1. Kac diagram and double Vogan diagram of $\mathfrak{s l}(2 n, \mathbb{R})$.

We want to obtain pseudo-Hermitian symmetric pairs $\left(\mathfrak{g}, \mathfrak{g}^{\sigma}\right)$. By Theorem 1.1(b), we circle the black vertex and another white vertex $\alpha$ where $m_{\alpha}=n_{\alpha}=1$. The two leftmost white vertices satisfy this condition. They are located in symmetric positions, so they lead to isomorphic $\mathfrak{g}^{\sigma}$. We circle one of them and obtain Figure 1(b), which represents $\mathfrak{g}^{\sigma}=\mathfrak{s l}(n, \mathbb{C})+\mathfrak{s o}(2)$. It appears as Figure $4(1)$ of the classification in the next section.

For exceptional real Lie algebras, we use Cartan's notation $\mathfrak{e}_{n(m)}$ (see for instance $[4$, Ch.X-6]) to denote the real form of $E_{n}$ with $m=\operatorname{dim} \mathfrak{p}-\operatorname{dim} \mathfrak{k}$.

Example 3.2. Pseudo-Hermitian symmetric pairs of $\mathfrak{e}_{6(2)}$. 


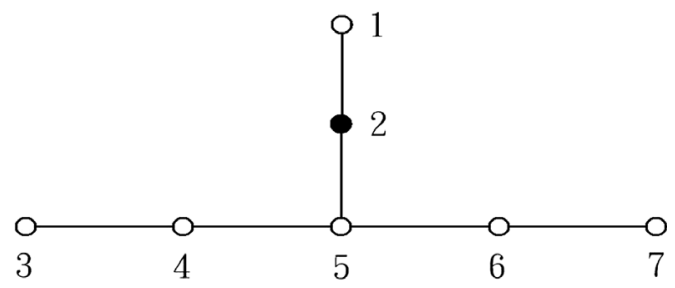

FiguRE 2. Kac diagram of $\mathfrak{e}_{6(2)}$.

For $\mathfrak{g}=\mathfrak{e}_{6(2)}$, the Kac diagram is given by Figure 2 above. We assign the numbers $1,2, \ldots, 7$ to the vertices for easy reference.

The white vertices form $\mathrm{D}_{\mathfrak{k}}=A_{1} \times A_{5}$. We consider all possible circlings such that at most one vertex from each connected component of $D_{\mathfrak{k}}$ is circled, and the black vertex may or may not be circled. We then use the equivalence relation in Definition 2.6 to omit redundant circlings and obtain the following four double Vogan diagrams [3, p.1743]:

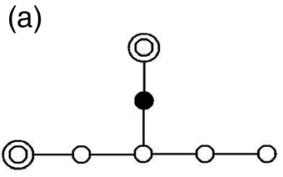

(b)

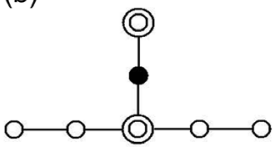

(c)

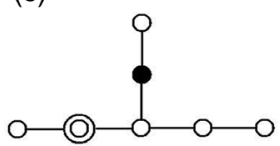

(d)

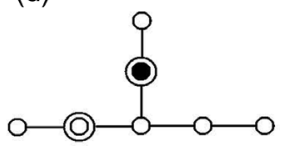

Figure 3. Double Vogan diagrams of $\mathfrak{e}_{6(2)}$.

These four diagrams represent four symmetric pairs. We next determine which ones are pseudo-Hermitian.

Let $\left(i_{1}, \ldots, i_{r}\right)$ denote the circling of vertices $i_{1}, \ldots, i_{r}$. For example Figure 3(a) is $(1,3)$. We have $m_{1}=m_{3}=m_{7}=1, m_{2}=m_{4}=m_{6}=2$ and $m_{5}=3$ [5, Ch.4]. By Theorems 2.1(a) and 2.7, each circling in Figure 3 is equivalent (in the sense of Definition 2.6) to one of the following circlings:

(a) $\quad\{$ circled vertices $\}=\{\alpha, \beta\}, m_{\alpha}=m_{\beta}=1$;

(b) $\quad$ circled vertices $\}=\{\alpha\}, m_{\alpha}=2$.

By Theorem 1.1(a), a circling is equivalent to (3.1)(a) if and only if the symmetric pair is pseudo-Hermitian.

Figure 3(a):

The circling $(1,3)$ already belongs to $(3.1)(\mathrm{a})$, so it represents a pseudo-Hermitian symmetric pair.

Figure 3(b):

Underline vertex $\alpha$ to denote the operation $F_{\alpha}$ of $(2.2)$. We have $(\underline{1}, 5) \rightarrow(1, \underline{2}, 5) \rightarrow$ $(2)$, so $(1,5)$ is equivalent to $(2)$. Since $(2)$ belongs to $(3.1)(b)$, it follows that $(1,5)$ does not represent a pseudo-Hermitian symmetric pair. 
Figure 3(c):

The circling (4) belongs to $(3.1)(\mathrm{b})$, so it does not represent a pseudo-Hermitian symmetric pair.

Figure 3(d):

We have $(2, \underline{4}) \rightarrow(2,3,4, \underline{5}) \rightarrow(3,5, \underline{6}) \rightarrow(3,6, \underline{7}) \rightarrow(3,7)$, so $(2,4)$ is equivalent to $(3,7)$. Since $(3,7)$ belongs to $(3.1)(\mathrm{a})$, it follows that $(2,4)$ represents a pseudoHermitian symmetric pair.

We conclude that Figures 3(a) and (d) represent pseudo-Hermitian symmetric pairs, while Figures 3(b) and (c) do not. Figures 3(a) and (d) appear as Figure 5(1,2) of the classification in the next section.

\section{Classification}

Using the method illustrated in the previous section, we apply Theorem 1.1 to classify all the irreducible pseudo-Hermitian symmetric pairs $\left(\mathfrak{g}, \mathfrak{g}^{\sigma}\right)$. We list them with their double Vogan diagrams in Figures 4 and 5. They match the list in [7, p.547-548].

Classical irreducible pseudo-Hermitian symmetric pairs

1. $(\mathfrak{s l}(2 n, \mathbb{R}), \mathfrak{s l}(n, \mathbb{C})+\mathfrak{s o}(2))$

2. $(\mathfrak{s u} *(2 n), \mathfrak{s l}(n, \mathbb{C})+\mathfrak{s o}(2))$

3. $(\mathfrak{s u}(n-i, i), \mathfrak{s}(\mathfrak{u}(h, k)+\mathfrak{u}(n-i-h, i-k)))$

4. $\left(\mathfrak{s o}^{*}(2 n), \mathfrak{s o}^{*}(2 n-2)+\mathfrak{s o}(2)\right)$

5. $(\mathfrak{s o *}(2 n), \mathfrak{u}(n-k, k))$

6. $(\mathfrak{s o}(n-k, k), \mathfrak{s o}(n-k, k-2)+\mathfrak{s o}(2))$

7. $(\mathfrak{s o}(2(n-k), 2 k), \mathfrak{u}(n-k, k))$

8. $(\mathfrak{s p}(n-i, i), \mathfrak{u}(n-i, i))$

In Figure 4(6), where $\mathfrak{g}=\mathfrak{s o}(n-k, k)$, the white and black circled vertices are adjacent. Both endpoints of the diagram can be of type $B$ or $D$. An odd (resp. even) parity among $\{n-k, k\}$ leads to a type $B$ (resp. type $D$ ) endpoint. For example if $n$ and $k$ are even, then both endpoints are of type $D$.

Exceptional irreducible pseudo-Hermitian symmetric pairs

1. $\left(\mathfrak{e}_{6(2)}, \mathfrak{s o}^{*}(10)+\mathfrak{s o}(2)\right)$

2. $\left(\mathfrak{e}_{6(2)}, \mathfrak{s o}(6,4)+\mathfrak{s o}(2)\right)$

3. $\left(\mathfrak{e}_{6(-14)}, \mathfrak{s o}(8,2)+\mathfrak{s o}(2)\right)$

4. $\left(\mathfrak{e}_{6(-14)}, \mathfrak{s o}^{*}(10)+\mathfrak{s o}(2)\right)$

5. $\left(\mathfrak{e}_{7(7)}, \mathfrak{e}_{6(2)}+\mathfrak{s o}(2)\right)$

6. $\left(\mathfrak{e}_{7(-5)}, \mathfrak{e}_{6(-14)}+\mathfrak{s o}(2)\right)$

7. $\left(\mathfrak{e}_{7(-5)}, \mathfrak{e}_{6(2)}+\mathfrak{s o}(2)\right)$

8. $\left(\mathfrak{e}_{7(-25)}, \mathfrak{e}_{6(-14)}+\mathfrak{s o}(2)\right)$ 
$\mathrm{O}_{1}^{\mathrm{O}} \mathrm{O}-\mathrm{a} \leftarrow \mathrm{O}$
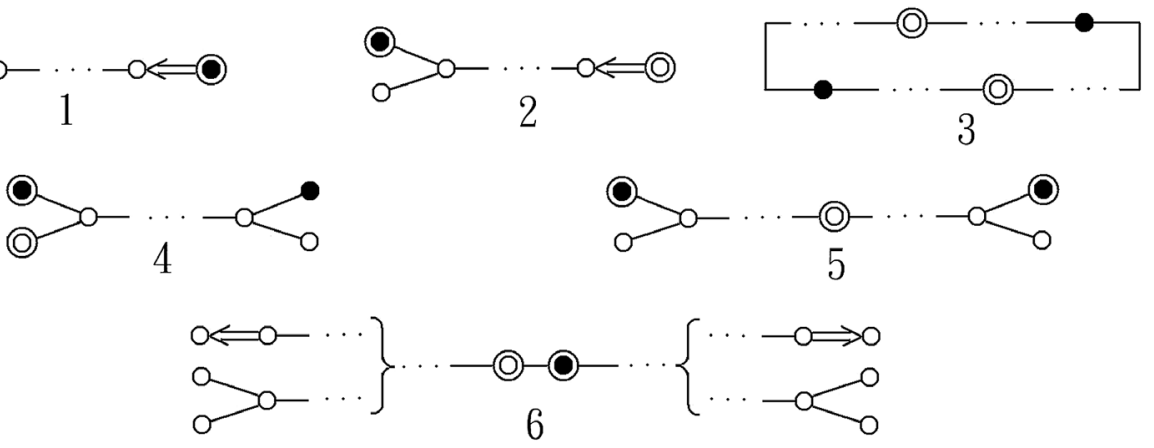

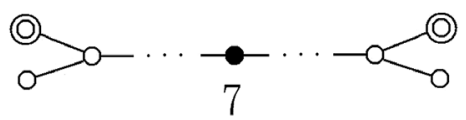

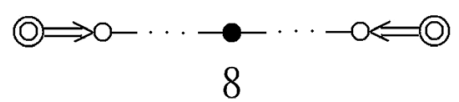

Figure 4. Classical irreducible pseudo-Hermitian symmetric pairs.<smiles>CC(OO)(OO)OO</smiles><smiles>OOC(OO)OO</smiles><smiles>COC(O)OO</smiles>

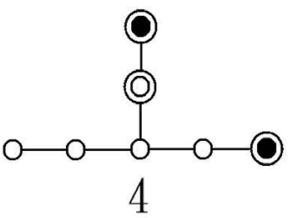<smiles>OOOC(O)OOO</smiles><smiles>O=COC(O)OOO</smiles><smiles>OOOOC(O)OOO</smiles><smiles>O=C(O)OOOO</smiles>

FiguRE 5. Exceptional irreducible pseudo-Hermitian symmetric pairs.

\section{Acknowledgments}

This work was supported in part by the National Science Council of Taiwan.

\section{References}

[1] M. Berger, Les espaces symétriques non compacts, Ann. Sci. École Norm. Sup. (3) 74 (1957), 85-177.

[2] M.K. Chuah and C.C. Hu, Extended Vogan diagrams, J. Algebra 301 (2006), 112-147.

[3] M.K. Chuah and J.S. Huang, Double Vogan diagrams and semisimple symmetric spaces, Trans. Amer. Math. Soc. 362 (2010), 1721-1750.

[4] S. Helgason, Differential Geometry, Lie Groups, and Symmetric Spaces, Graduate Studies in Math. 34, Amer. Math. Soc., Providence, 2001. 
[5] V.G. Kac, Infinite Dimensional Lie Algebras, 3rd ed., Cambridge University Press, Cambridge, 1990.

[6] A.W. Knapp, Lie Groups beyond an Introduction, 2nd ed., Progr. Math. Vol. 140, Birkhäuser, Boston, 2002.

[7] R.A. Shapiro, Pseudo-Hermitian symmetric spaces, Comment. Math. Helv. 46 (1971), 529-548.

Department of Mathematics, National Tsing Hua University, Hsinchu 300, Taiwan

E-mail address: chuah@math.nthu.edu.tw 
